\title{
Tecnura
}

\section{Construcción y evaluación de un divisor de alto voltaje para pruebas de impulso tipo rayo}

\section{Construction and evaluation of a high voltage divider for lightning impulse tests}

\author{
Juan David Mina-Casaran¹, Diego Fernando García², Diego Fernando Echeverry
}

Fecha de recepción: 5 de febrero de 2019

Fecha de aceptación: 15 de mayo de 2019

Cómo citar: Mina-Casaran, J.D., García, D.F. y Echeverry, D.F. (2019). Construcción y evaluación de un divisor de alto voltaje para pruebas de impulso tipo rayo. Tecnura, 23(61), 23(61), 31-44. doi: https://doi. org/10.14483/22487638.14385

\section{Resumen}

Contexto: La medición segura y efectiva de señales de alto voltaje se ha convertido, desde hace años, en una de las mayores preocupaciones por parte de quienes realizan ensayos en alta tensión. En ese sentido, el divisor de tensión representa uno de los dispositivos de mayor importancia para la medición en laboratorio de señales como las generadas por los rayos. Sin embargo, el divisor debe contar con características adecuadas de voltaje nominal, factor de escala y respuesta en frecuencia, para que la señal registrada sea un reflejo de la señal aplicada al equipo bajo prueba. Muestra de ello son los tipos más comunes de divisores de tensión desarrollados (divisor resistivo, divisor capacitivo y divisor capacitivo amortiguado), cada uno con características propias que permiten un buen desempeño del sistema de medición y registro en alta tensión. Con el propósito de evaluar el desempeño del sistema de medición y registro, en lo referente a la etapa de conversión, en este trabajo se presentan los aspectos teóricos y prácticos relacionados con el diseño, construcción y evaluación de un divisor capacitivo amortiguado de 300 kV, usado para la medición de señales de impulso de tensión tipo rayo.

Método: Para el diseño y evaluación del divisor de voltaje, se utilizaron herramientas computacionales de simulación, como Mathematica ${ }^{\circledR}$ y Pspice ${ }^{\circledR}$, las cuales ayudaron a estimar el comportamiento de los componentes del divisor: resistor de amortiguamiento y ramas de bajo voltaje. Del mismo modo, se utilizaron equipos de laboratorio (medidor de respuesta en frecuencia y generador de impulsos de voltaje de $300 \mathrm{kV}$ ), para verificar el desempeño de los componentes del divisor. Los ensayos se basaron en pruebas de respuesta en frecuencia, capacidad de aislamiento y obtención de los factores de escala del divisor, de acuerdo con estándares internacionales.

Resultados: En la prueba de respuesta en frecuencia realizada al resistor de amortiguamiento, desde $20 \mathrm{~Hz}$ hasta aproximadamente $1 \mathrm{MHz}$, los efectos parásitos son despreciables y la impedancia del resistor se puede considerar puramente resistiva. Por

1 Ingeniero electricista, magíster en Ingeniería Eléctrica. Estudiante de Doctorado en Ingeniería del Grupo de Investigación en Alta Tensión (Gralta), Universidad del Valle. Cali, Colombia. Contacto: juan.mina@correounivalle.edu.co ORCID: http://orcid.org/0000-0002-9670-6897

2 Ingeniero electricista, magíster de Generación de Energía Eléctrica, doctor en Ingeniería. Docente del Grupo de Investigación en Alta Tensión (Gralta), Universidad del Valle. Cali, Colombia. Contacto: diego.garcia@correounivalle.edu.co ORCID: https://orcid.org/0000-00031055-1415

3 Ingeniero electricista, doctor en Ingeniería de la Universidad del Valle. Docente del Grupo de Investigación en Alta Tensión (Gralta), Universidad del Valle. Cali, Colombia. Contacto: diego.echeverry@correounivalle.edu.co ORCID: http://orcid.org/0000-0002-6067-2955 
su parte, en la prueba de capacidad de aislamiento, se observó que el resistor puede soportar impulsos de tensión de hasta $10 \mathrm{kV}$, sin que se produzca ruptura del aislamiento del resistor. Respecto a la verificación de los factores de escala, las diferencias porcentuales no superaron el límite de variación de voltaje pico establecido por norma.

Conclusiones: Los resultados muestran que la metodología desarrollada fue adecuada para el diseño, construcción y simulación del divisor de tensión. Los modelos eléctricos propuestos en la metodología fueron suficientes para obtener resultados confiables en las simulaciones. Finalmente, las contribuciones más importantes del trabajo fueron la construcción de un resistor de amortiguamiento no inductivo y la construcción de una rama adicional de bajo voltaje.

Palabras clave: divisor de voltaje, factor de escala, impulso de tensión, rama de bajo voltaje, resistor de amortiguamiento.

\section{Abstract}

Context: For years, the safe and effective measurement of high voltage signals has one of the major concerns of those conducting high voltage tests; in these cases, voltage divider represents one of the most important devices for laboratory measurement of signals (such as those generated by lightning). However, the divider must have adequate characteristics of nominal voltage, scale factor, and frequency response, so that the recorded signal is a reflection of the signal applied to the equipment under test. For example, the most commonly developed voltage dividers (resistive divider, capacitive divider, and damped capacitive divider) have their own characteristics that allow a good performance of the high voltage measurement and recording system. This paper presents the theoretical and practical aspects related to design, construction, and evaluation of a damped capacitive divider of $300 \mathrm{kV}$ in order to evaluate the performance of the measurement and recording system in relation to the conversion stage.

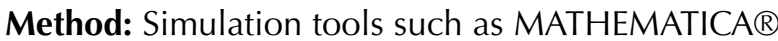
and PSPICE $®$ were used for the design and evaluation of the voltage divider. These helped estimate the behavior of the divider components (damping resistor and low voltage arms). Additionally, laboratory equipment (frequency response meter and $300 \mathrm{kV}$ voltage pulse generator) was used to verify the performance of the components. The experiments were based on frequency response tests, insulation capacity, and obtaining the scale factors according to national and international standards.

Results: In the frequency response test performed on the damping resistor from $20 \mathrm{~Hz}$ to $1 \mathrm{MHz}$, the parasitic effects are negligible and the impedance of the resistor can be considered purely resistive. In the insulation capacity test, resistor can withstand voltage pulses of up to $10 \mathrm{kV}$ without breaking the resistor insulation. Regarding the verification of the scale factors, the porcentage differences did not exceed the limit of peak voltage variation set by the standard.

Conclusions: The results show the methodology developed was adequate for the design, construction, and simulation of the voltage divider. The electrical models suggested in the methodology were sufficient to obtain reliable results during simulations. Finally, the most important contributions of this work were the construction of a non-inductive damping resistor and the construction of an additional low-voltage branch.

Keywords: damping resistor, lightning impulse voltage, low voltage branch, scaling factor, voltage divider.

\section{INTRODUCCIÓN}

Los sistemas de medición para ensayos de alta tensión en laboratorio incorporan dispositivos de conversión, transmisión y registro de datos. Una de las partes más importantes del proceso, la tiene el dispositivo de conversión, conocido como divisor de tensión, el cual reduce la amplitud del voltaje a un valor adecuado y seguro para los dispositivos de medición y registro (Pattarakijkul, Kurupakorn y 
Charoensook, 2010). Los divisores de tensión han sido ampliamente utilizados en diversas aplicaciones con el propósito de garantizar procesos de medición seguros de señales de alto voltaje (Daum y Lynch, 1989; Kovačević, Brajović, Stanković y Osmokrović, 2016; Ortiz, Jiménez y Ramos, 2014). En los ensayos de impulso de tensión tipo rayo, existen dos dificultades relacionadas con la medición de la señal (García, Saens, Prado y Martinez, 2009): i) la gran amplitud de dichas señales, que se encuentra en el orden de kilovoltios hasta megavoltios, y ii) sus pendientes de variación rápida, las cuales están dentro del rango de décimas de microsegundos. Por estas razones, el divisor debe contar con valores adecuados de voltaje nominal, factor de escala y respuesta en frecuencia, para que la señal registrada sea un reflejo de la señal aplicada al equipo bajo prueba.

Los divisores de tensión empleados para medición de señales de impulso pueden clasificarse en tres tipos según los elementos pasivos usados y la configuración de las ramas de alto y bajo voltaje (Kuffel, Zaengl y Kuffel, 2000; Passon, Gitin y Meisner, 2016; Teachawong y Trakulkan, 2014; Yan, Zhao y Li, 2018): resistivos, capacitivos y capacitivos amortiguados.

En su estructura, un divisor resistivo tiene dos ramas resistivas conectadas en serie. Este tipo de divisor se caracteriza por presentar un excelente tiempo de respuesta. Sin embargo, cuando la resistencia total es alta, el divisor presenta una característica de transferencia desfavorable y cuando la resistencia total es baja, se afecta la forma de la señal aplicada. Con respecto al factor de escala, este parámetro es dependiente de la frecuencia de la señal dada la presencia de capacitancias parásitas.

Los divisores capacitivos están conformados por dos ramas capacitivas y representan una mejora respecto a los divisores resistivos, ya que eliminan la dependencia del factor de escala con la frecuencia (Kuffel, Zaengl y Kuffel, 2000; García et al., 2009; Prochazka, Hlavacek, Knenicky y Mahmoud, 2016). La ubicación del divisor en el recinto de pruebas y la impedancia del instrumento de medida que queda en paralelo con la capacitancia de la rama de bajo voltaje del divisor provocan oscilaciones y variaciones en la señal de salida, lo cual dificulta su medida. Los divisores capacitivos amortiguados reducen dichas oscilaciones mediante la incorporación de resistores de amortiguamiento en las ramas de alto o bajo voltaje. No obstante, este tipo de divisor para impulso sigue presentando algunas dificultades relacionadas con inductancias parásitas, capacitancias parásitas y capacitancias distribuidas (Kuffel, Zaengl y Kuffel, 2000). Las inductancias parásitas surgen por las conexiones entre los elementos pasivos que conforman las ramas de alto y bajo voltaje, causando oscilaciones en el frente de la señal medida y retardo del tiempo de respuesta del divisor. Las capacitancias parásitas obedecen a la disposición y geometría resultante de los elementos que conforman las ramas de alto y bajo voltaje del divisor. Dichas capacitancias atenúan la señal medida, lo que dificulta su registro en los dispositivos utilizados para tal fin. Por su parte, en el momento de la aplicación del impulso, entre el borne de entrada de la señal y tierra aparece una capacitancia distribuida que provoca grandes esfuerzos eléctricos entre los elementos pasivos que constituyen la rama superior del divisor (Teachawong y Trakulkan, 2014). En la práctica solo puede ser parcialmente compensada con la instalación de electrodos relajadores de campo eléctrico (Pattanadech, Potivetkul y Yuttagowith, 2006).

En este artículo se presenta una metodología con los requerimientos necesarios para el diseño, construcción y evaluación de un divisor de tensión capacitivo amortiguado (DCA) de 300 kV, utilizado para medición de señales de impulso tipo rayo $(1,2 / 50 \mu \mathrm{s}$, según norma) (Alfonso, Perdomo, Santamaria y Gómez, 2014; García y Rincón, 2009). El artículo está dividido de la siguiente forma: inicialmente se presenta el DCA de $300 \mathrm{kV}$ previo, utilizado como insumo y del cual se identificaron posibles mejoras en algunos de sus componentes. Luego se muestra la metodología utilizada y sus etapas. Posteriormente, se exponen los resultados 
de los ensayos realizados con base en procedimientos descritos en estándares internacionales IEEE Standard for High-Voltage Testing Techniques (2013) e IEC High-voltage Test Techniques, Part 2: Measuring Systems (2010). Por último, se presentan las conclusiones principales del trabajo.

\section{DIVISOR DE TENSIÓN CAPACITIVO AMORTIGUADO PARA IMPULSO}

El modelo eléctrico simplificado de un divisor de tensión capacitivo amortiguado, se muestra en la figura 1. Este se compone de dos ramas capacitivas de alto y bajo voltaje (C.A.V, C.B.V) conectadas en serie con una resistencia de amortiguamiento $(R d a)$ y una inductancia parásita $(L p)$. El modelo también incluye una capacitancia parásita $(C p)$. Los valores Vi_n y Vo_n representan los voltajes de entrada y salida del divisor.

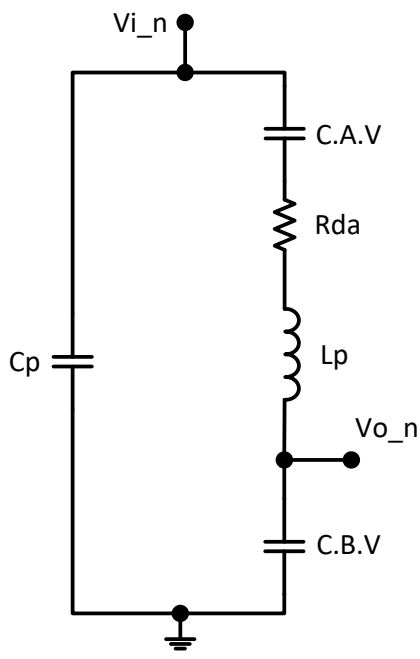

Figura 1. Modelo eléctrico simplificado del DCA

Fuente: elaboración propia.

Por otra parte, para que el divisor pueda medir adecuadamente las señales de impulso, debe cumplir con los requerimientos eléctricos descritos a continuación:
- Voltaje nominal y voltaje máximo. Son el valor nominal para el cual ha sido previsto el funcionamiento del divisor, y el voltaje máximo que puede alcanzar el divisor sin que represente un riesgo en su operación.

- Factor de escala. Es la relación entre las tensiones nominales de entrada y salida del divisor. Del modelo eléctrico simplificado presentado anteriormente, al despreciar los efectos parásitos se obtiene el factor de escala (a), tal como se muestra en la ecuación (1):

$$
a=\frac{V_{i_{-} n}}{V_{o_{-} n}}=\frac{C_{B . V} \cdot\left(1+R_{d a} \cdot C_{A, V}\right)}{C_{A . V}}
$$

- Cargabilidad. Para que el divisor de tensión no influya de manera significativa en la forma de la señal aplicada, su capacitancia total debe ser como mínimo 10 veces menor a la capacitancia del generador de impulso.

- Tiempo de respuesta. Hace referencia al comportamiento dinámico del divisor de tensión ante una entrada tipo escalón unitario.

- Ancho de banda. Indica la máxima frecuencia que el divisor puede tolerar sin que se produzcan recortes en la señal medida. Para que no existan recortes en el frente de la señal medida, el ancho de banda del divisor debe ser mayor a 1,2 $\mathrm{MHz}$.

- Distribución lineal del campo eléctrico. Las capacitancias parásitas provocan grandes esfuerzos eléctricos entre los condensadores de la rama superior del divisor. Dichos esfuerzos se reducen en la práctica con la instalación de electrodos relajadores de campo eléctrico.

\section{Aspectos generales del DCA de $300 \mathrm{kV}$}

El prototipo de DCA de $300 \mathrm{kV}$ se muestra en la figura 2. El divisor es utilizado para pruebas de impulso de tensión tipo rayo en el Laboratorio de Alta Tensión (LAT) de la Universidad del Valle. 


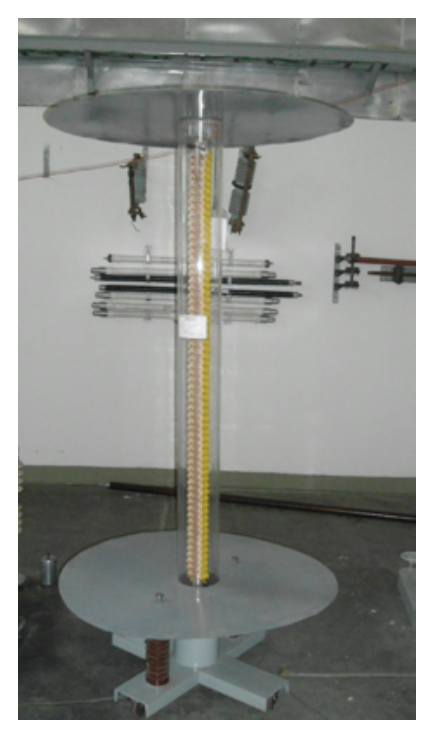

Figura 2. Divisor de tensión capacitivo amortiguado del LAT

Fuente: Laboratorio de Alta Tensión. Universidad del Valle.

Se compone por las ramas de alto y bajo voltaje, electrodos relajadores de campo eléctrico, tubo soporte (cuerpo del divisor) y plataforma para su movilidad (Vera y Prado, 2006). La rama de alto voltaje posee 125 condensadores de $0,1 \mu \mathrm{F}$ y $3 \mathrm{kV}$ cada uno, conectados en serie a un resistor de amortiguamiento $R d a$. La rama inferior cuenta con una capacitancia equivalente de $0,68 \mu \mathrm{F}$. El resistor se compone de un cilindro de policroruro de vinilo (PVC) el cual aloja una solución líquida de sal y agua destilada. Por otra parte, para reducir los esfuerzos eléctricos entre los condensadores de la rama superior, el divisor emplea electrodos relajadores de campo eléctrico de placas paralelas.

Las especificaciones técnicas del divisor se muestran en la tabla 1 (Vera y Prado, 2006).

A continuación, se presentan las desventajas técnicas sobre el prototipo de DCA de 300 kV, las cuales dificultan su desempeño:

- Resistor de amortiguamiento líquido. Este tipo de resistor permite obtener diferentes valores de resistencia dependiendo de la cantidad de sal en el líquido, pero presenta desventajas como el
Tabla 1. Especificaciones técnicas del DCA de 300 kV inicial

\begin{tabular}{lc}
\hline Voltaje de entrada nominal & $300 \mathrm{kV}$ \\
\hline Voltaje de entrada máximo & $375 \mathrm{kV}$ \\
\hline Voltaje de salida nominal & $353 \mathrm{~V}$ \\
\hline Factor de escala & $850: 1$ \\
\hline Capacitancia de alto voltaje & $800 \mathrm{pF}$ \\
\hline Capacitancia de bajo voltaje & $0,68 \mu \mathrm{F}$ \\
\hline Resistencia de amortiguamiento & $475 \Omega$ \\
\hline Capacitancia parásita & $43 \mathrm{pF}$ \\
\hline Inductancia parásita & $35 \mu \mathrm{H}$ \\
\hline Ancho de banda & $929 \mathrm{kHz}$ \\
\hline Altura & $2,2 \mathrm{~m}$ \\
\hline Diámetro ext. de las placas (relajador de campo) & $1 \mathrm{~m}$ \\
\hline
\end{tabular}

Fuente: elaboración propia.

mantenimiento continuo que se debe realizar a la solución para garantizar el valor de resistencia. Por otra parte, dado el tipo de conducción presente en el resistor (por ionización), no existe un modelo definido que ayude a reducir los efectos inductivos presentes a altas frecuencias los cuales causan oscilaciones en el frente de la señal medida.

- Rama de baja tensión. El divisor cuenta con un factor de escala de 850:1, adecuado para impulsos de tensión entre 100 kV y 300 kV, pero para tensiones menores a $100 \mathrm{kV}$ (utilizadas normalmente en el LAT) se presentan problemas de ruido a la salida del divisor.

- Cuerpo del divisor de tensión. Se compone de un tubo de acrílico el cual presenta un espacio inadecuado para alojar el resistor de amortiguamiento.

\section{Propuestas de mejora sobre el prototipo de DCA de $300 \mathrm{kV}$}

Para dar solución a los problemas mencionados, en este trabajo se propuso: 
- Mitigar las oscilaciones que se presentan a la salida del divisor por medio de la construcción de un resistor de baja inductancia a base de alambre de níquel y cromo.

- Adicionar una rama de bajo voltaje con factor de escala menor a 850:1 que garantice una mayor exactitud en la medición de señales menores a $100 \mathrm{kV}$, sin influencia de ruido.

- Cambiar el cuerpo del divisor con el propósito de darle mayor rigidez mecánica y proporcionar el espacio adecuado para alojar el resistor de amortiguamiento.

\section{METODOLOGÍA DE DISEÑO, CONSTRUCCIÓN Y EVALUACIÓN DEL DCA}

La metodología propuesta para el diseño, construcción y evaluación del DCA de 300 kV requiere el desarrollo de tres etapas las cuales se presentan a continuación (figura 3).

\section{Identificación de parámetros previos de diseño}

En esta etapa se presenta el cálculo de los parámetros de diseño del DCA de $300 \mathrm{kV}$ para medición de ondas de impulso de 1,2/50 $\mu$ s.

\section{Determinación del número de condensadores}

de la rama de alto voltaje

El número mínimo de condensadores de la rama de alto voltaje (nmín) se determina a partir de la ecuación (2):

$$
n_{\min }=\frac{V_{\max }}{V_{n c}}
$$

Donde, Vmax y Vnc son los voltajes máximo y nominal del divisor y para cada condensador, respectivamente.

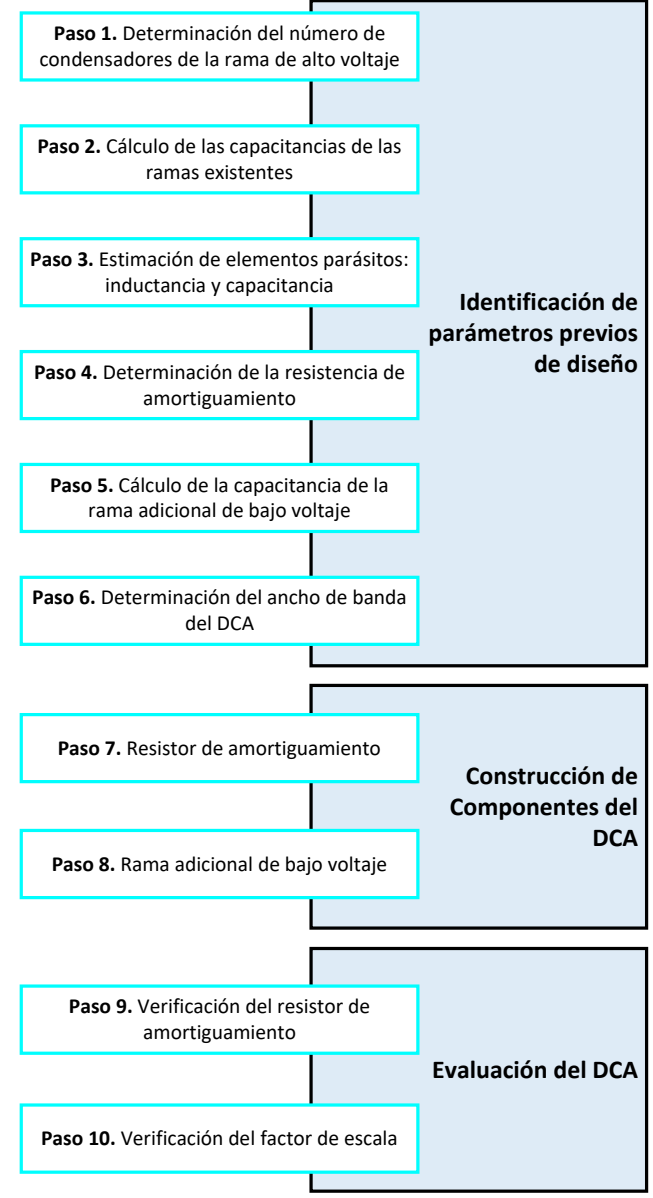

Figura 3. Metodología para el diseño, construcción y evaluación de un DCA de 300 kV

Fuente: elaboración propia.

\section{Cálculo de las capacitancias de las ramas existentes}

Las capacitancias C.A.V y C.B.V se determinan de acuerdo con las ecuaciones (3) y (4):

$$
\begin{gathered}
C_{A . V}=\frac{C_{A . V \_} i}{n} \\
C_{B . V}=\frac{C_{A . V}}{a}
\end{gathered}
$$


Donde, C.A.B_i es la capacitancia del condensador $i$, igual para todos los condensadores, $n$ representa el número total de condensadores conectados en serie en la rama de alto voltaje, y a es el factor de escala del divisor.

Estimación de elementos parásitos: inductancia y capacitancia

Para el cálculo de la capacitancia parásita, se puede utilizar la ecuación descrita en la ecuación (5) y propuesta en Kuffel, Zaengl y Kuffel (2000).

$$
C_{p}=2 \pi \varepsilon_{0} \frac{l_{1}+l_{2}+l_{3}}{3},[F]
$$

Donde, I1, I2 y I3 son la altura, largo y ancho del divisor. El parámetro عo constituye la permitividad del aire.

Determinación de la resistencia de

amortiguamiento

Según Kuffel, Zaengl y Kuffel (2000), el valor de la resistencia de amortiguamiento debe ser entre dos y cuatro veces el valor de impedancia característica del divisor. El rango de variación de Rda se muestra en la ecuación (6).

$$
2 \sqrt{\frac{L_{p}}{C_{A . V}+C_{p}}} \leq R_{d a} \leq 4 \sqrt{\frac{L_{p}}{C_{A . V}+C_{p}}}
$$

Para encontrar el valor adecuado de Rda es necesario simular el comportamiento del divisor de tensión empleando el modelo equivalente de la figura 1. El análisis se inicia utilizando el valor mínimo de resistencia y se modifica dentro del rango estipulado, hasta obtener una señal de salida correspondiente a la señal de impulso aplicada.

Cálculo de la capacitancia de la rama adicional de bajo voltaje

Para determinar la capacitancia equivalente de la rama adicional de bajo voltaje se utiliza la ecuación (1), y se consideran los siguientes parámetros de diseño:

- $50 \%$ de la tensión nominal del divisor.

- Factor de escala: (413:1).

- Voltaje de salida del divisor: Vi_n/a.

- Capacitancia de la rama de alta tensión: C.A.V.

- Resistencia de amortiguamiento: Rda.

Determinación del ancho de banda del DCA

Para determinar el ancho de banda del divisor, se emplea la herramienta computacional

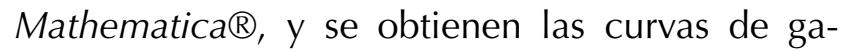
nancias del divisor en función de las frecuencias $\omega$ (radianes) y $\sigma$ (nepers), a partir de su función de transferencia mostrada en la ecuación (7).

$$
H(s)=\frac{V_{o_{-} n}}{V_{i_{-} n}}=\frac{k_{0}}{k_{1} s^{2}+k_{2} s+k_{3}}
$$

Donde, $k 0, k 1, k 2, k 3$ son constantes que dependen de los valores de los elementos pasivos del divisor. El DCA cuenta con funciones de transferencia $H 1$ (s) y $H 2(s)$ dados los factores a1 (413:1) y a2 (850:1).

Las superficies de ganancia del divisor para cada factor de escala, interceptan los planos 1/413 (figura 4) y 1/850 (figura 5).

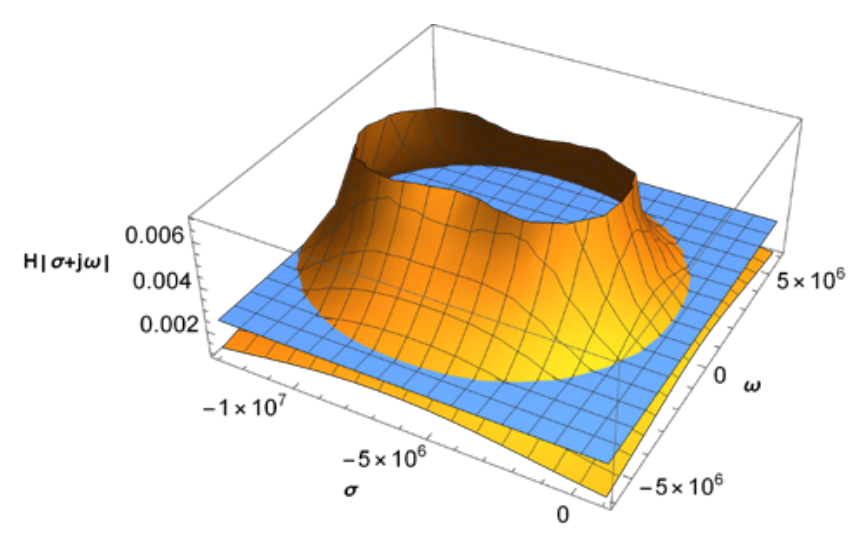

Figura 4. Intersección del plano de ganancia 1/413 del divisor y su superficie de respuesta en frecuencia H1(s)

Fuente: elaboración propia. 


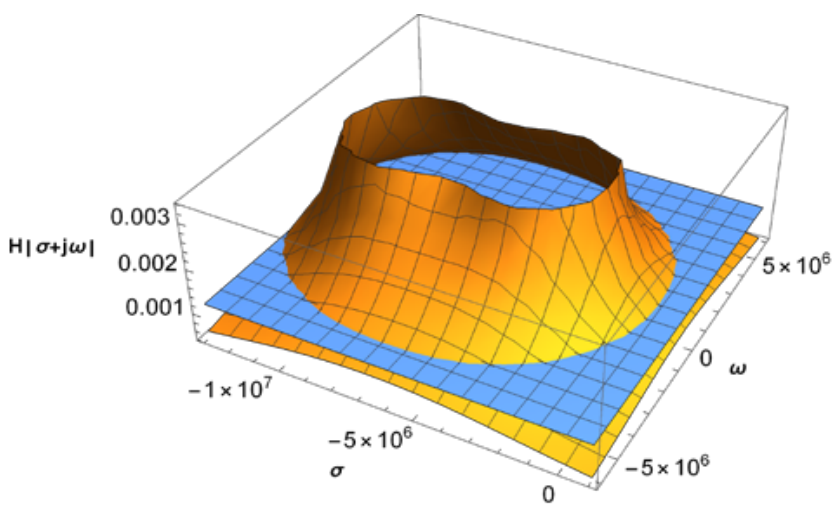

Figura 5. Intersección del plano de ganancia 1/850 del divisor y su superficie de respuesta en frecuencia $\mathrm{H} 2(\mathrm{~s})$

Fuente: elaboración propia.

Los puntos extremos de la intersección de las dos superficies corresponden a las frecuencias de corte $\omega$ y $\sigma$. Por su parte, la figura 6 muestra la proyección de las intersecciones de los planos 1/413 y $1 / 850$ con las superficies de respuesta en frecuencia $H 1(s)$ y $H 2(s)$, vistas desde los ejes $\omega$ y $\sigma$, y que además delimitan el ancho de banda del DCA. Las frecuencias neperianas $\sigma 1$ y $\sigma 2$ representan los límites máximos de frecuencias del divisor. En ese sentido, el ancho de banda $A B$ se calcula como se presenta en la ecuación (8):

$$
A B=\sigma_{1}-\sigma_{2}
$$

\section{Construcción de componentes del DCA}

En esta etapa se presenta el proceso de construcción de los componentes que se diseñaron para lograr un mejor desempeño del DCA.

\section{Resistor de amortiguamiento}

El resistor fue diseñado para valores transitorios de voltaje y corriente de $10 \mathrm{kV}$ y 29 A, los cuales se obtuvieron al simular el sistema de generación y medición de un impulso de tensión de 300 kV por medio de la herramienta computacional Pspice ${ }^{\circledR}$ (Mina, 2013). Para la construcción del resistor se empleó un alambre de níquel y cromo. La técnica aplicada consistió en arrollar en forma de vaivén, el alambre alrededor de un cilindro de PVC (figura 7), con el objeto de minimizar los campos magnéticos en toda la longitud del resistor, cuando por el alambre circulara una corriente determinada. Este tipo de construcción se consigue sin necesidad de reducir la potencia y rendimiento del resistor y solo requiere la mitad del alambre que emplearía una configuración del tipo Ayrton-Perry (Pan et al., 2015).

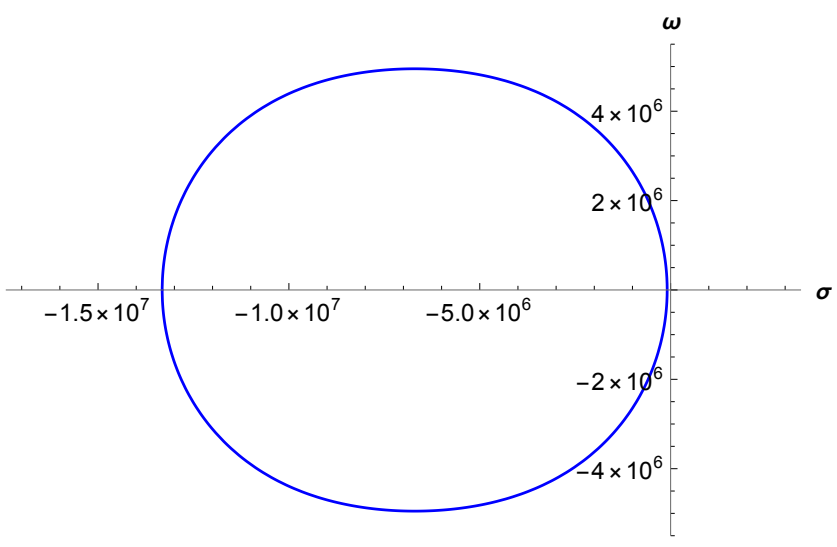

Figura 6. Proyección de las intersecciones de las ganancias del divisor con las superficies de respuesta en frecuencia $H 1$ (s) y $H 2$ (s) (vista superior)

Fuente: elaboración propia. 


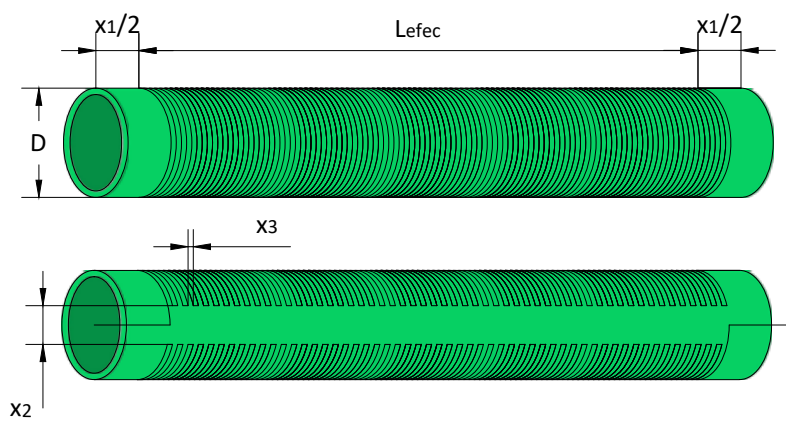

Figura 7. Disposición del alambre a lo largo del tubo de PVC

Fuente: elaboración propia.

El resistor fue alojado dentro de otro cilindro de PVC que posteriormente se llenó de aceite dieléctrico con el propósito de darle mayor capacidad de aislamiento. En la tabla 2 se presentan las especificaciones técnicas del resistor de amortiguamiento final.

Rama de bajo voltaje adicional

En la figura 8 se observan las ramas de bajo voltaje del divisor. Cada una cuenta con un arreglo serie-paralelo de 16 condensadores con capacitancia equivalente $0,33 \mu \mathrm{F}$ y $0,68 \mu \mathrm{F}$ respectivamente.

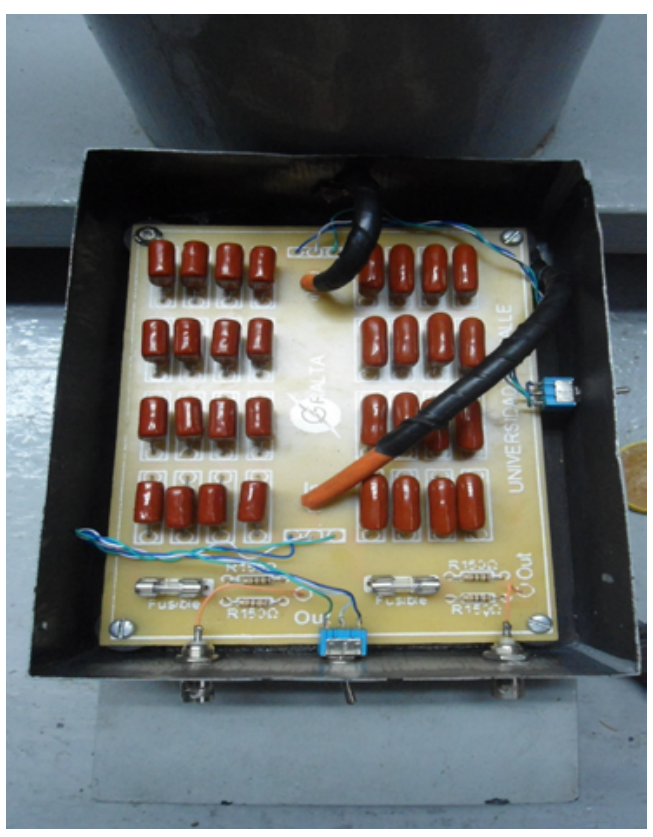

Figura 8. Ramas de baja tensión del divisor

Fuente: Laboratorio de Alta Tensión. Universidad del Valle.

Cada condensador soporta una tensión máxima de $400 \mathrm{~V}$ (1600 V por rama de baja tensión). Los resistores $R 1 a$ y $R 1 b$ (75 $\Omega$ cada uno) se utilizan para mitigar el efecto de onda viajera de la señal de impulso que se genera en el cable coaxial conectado entre el divisor y el osciloscopio.

Tabla 2. Especificaciones técnicas del resistor de amortiguamiento final

\begin{tabular}{lll}
\hline Resistencia por unidad de longitud & Ral & $34,8 \Omega / \mathrm{m}$ \\
\hline Longitud del alambre & Lal & $13,7 \mathrm{~m}$ \\
\hline Longitud del tubo soporte del alambre & $\mathrm{L}$ & $0,3 \mathrm{~m}$ \\
\hline Espacio de seguridad & $\mathrm{x} 1$ & $0,04 \mathrm{~m}$ \\
\hline Longitud efectiva del tubo & Lefec=L-x1 & $0,26 \mathrm{~m}$ \\
\hline Diámetro del tubo & $\mathrm{D}$ & $0,05 \mathrm{~m}$ \\
\hline Perímetro del tubo & $\mathrm{P}$ & $0,16 \mathrm{~m}$ \\
\hline Separación entre principio y final de cada espira & $\mathrm{x} 2$ & $0,018 \mathrm{~m}$ \\
\hline Perímetro efectivo del tubo & Pefec=P-x2 & $0,142 \mathrm{~m}$ \\
\hline Número de espiras & $\mathrm{E}=$ Lal/ Pefec & 97 \\
\hline Separación entre espiras & x3=Lefec/E & $0,0026 \mathrm{~m}$ \\
\hline Voltaje de cada espira & Vesp=10 kV/E & $103 \mathrm{~V}$ \\
\hline Voltaje entre espiras & Ve_e=2.Vesp & $206 \mathrm{~V}$ \\
\hline Resistencia esperada & $\mathrm{R}=\mathrm{E}$. Pefec.Ral & $479 \Omega$ \\
\hline
\end{tabular}

Fuente: elaboración propia. 


\section{Evaluación del DCA}

La evaluación del divisor consistió en: i) verificación del funcionamiento del resistor de amortiguamiento a través de pruebas de respuesta en frecuencia y capacidad de aislamiento, y ii) verificación de los factores de escala del divisor de acuerdo con los procedimientos descritos en IEEE Standard for $\mathrm{Hi}$ gh-Voltage Testing Techniques (2013) y en IEC High-Voltage Test Techniques (2010).

\section{Verificación del funcionamiento del resistor de} amortiguamiento

El resistor de amortiguamiento fue evaluado mediante pruebas experimentales en el LAT. Se realizaron ensayos de respuesta en frecuencia, para determinar posibles efectos parásitos en el resistor a altas frecuencias. Por medio de la ecuación (9) se calcula la inductancia parásita para un determinado valor de frecuencia $\omega$. XRda es la reactancia del resistor para el valor de frecuencia dado.

$$
L_{R d a}=\frac{X_{R d a}}{\omega}
$$

Para verificar la capacidad de aislamiento del resistor, fueron aplicados entre sus terminales diversos impulsos de 10 kV. Para ello se utilizó un generador de impulsos de voltaje (GIV) de $300 \mathrm{kV}$ y un divisor de tensión resistivo relación 1000:1.

Verificación de los factores de escala del DCA Se realiza de acuerdo con lo establecido en la norma IEC 60060-2. El diagrama esquemático de la prueba se muestra en la figura 9. Para cada rama de baja tensión del divisor se aplicaron 24 impulsos de 1,2/50 us y magnitud entre 30 kV y 300 kV. Cada factor de escala experimental se calculó a partir de la ecuación (10).

$$
n_{i}=\frac{V_{o 3 \_i} .1000}{V_{o \__{-} i}}
$$

Donde, Vo3_i representa el dato $i$ de la señal de salida del divisor resistivo, Vox_ $i$ es el dato $i$ de la señal de salida del DCA: $x=1$ para el factor de escala a1, $x=2$ para el factor de escala a2.

Para verificar la validez de los factores de escala obtenidos experimentalmente, la norma IEC 60060-2 establece un margen máximo de

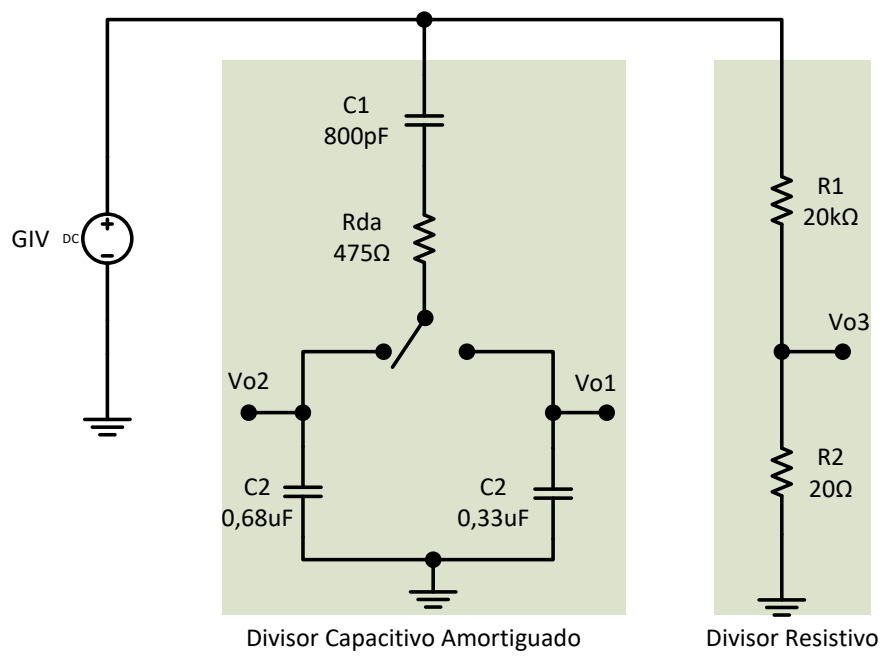

Figura 9. Esquema de prueba para la evaluación del DCA

Fuente: elaboración propia. 
diferencia de $\pm 3 \%$ entre los voltajes máximos de las señales de impulso del divisor bajo prueba y el divisor utilizado como patrón o referencia. Por medio de la ecuación (11) se realiza el cálculo de la diferencia porcentual, donde V1_i y V3_i son los voltajes de entrada para el ensayo $i$ de los divisores capacitivo amortiguado y resistivo.

$$
d i f_{\% \_i}=\left|\frac{V_{3 \_i}-V_{1 \_i}}{V_{3 \_i}}\right| .100
$$

\section{RESULTADOS Y ANÁLISIS}

A continuación, se presentan los resultados de las pruebas realizadas al DCA para comprobar su funcionamiento.

\section{Respuesta en frecuencia del resistor RDA}

En la figura 10 se observa el comportamiento de la impedancia y el ángulo de fase con relación a la frecuencia en el resistor de amortiguamiento. Desde $20 \mathrm{~Hz}$ hasta aproximadamente $1 \mathrm{MHz}$ los efectos

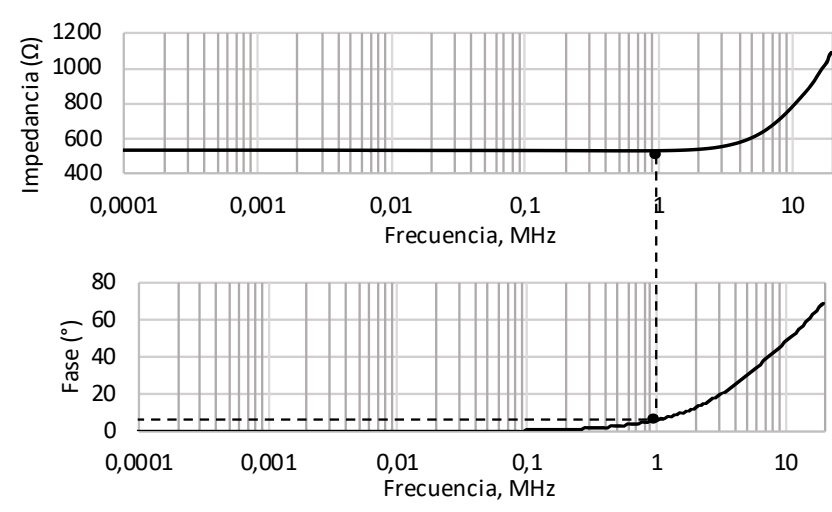

Figura 10. Gráfica de respuesta en frecuencia del resistor de amortiguamiento

Fuente: elaboración propia. parásitos son despreciables y la impedancia del resistor se puede considerar puramente resistiva.

A partir de la ecuación (9), se calculó la inductancia parásita presente en el resistor a 1,04 MHz. La impedancia del resistor y su inductancia parásita, para el valor de frecuencia dado, equivalen a:

$$
Z=(481+j 54) \Omega, L_{p}=8,3 \mu H
$$

\section{Capacidad de aislamiento del resistor RDA}

Los resultados obtenidos de los impulsos aplicados sobre el resistor de amortiguamiento (figura 11) indican una buena capacidad de aislamiento. El resistor puede soportar impulsos de tensión de hasta $10 \mathrm{kV}$, sin que se produzcan fallas en toda su estructura.

\section{Verificación de los factores de escala del DCA}

El comportamiento estadístico de los factores de escala calculados experimentalmente a partir de la ecuación (10), se muestran en las figuras 12 y 13 , respectivamente.

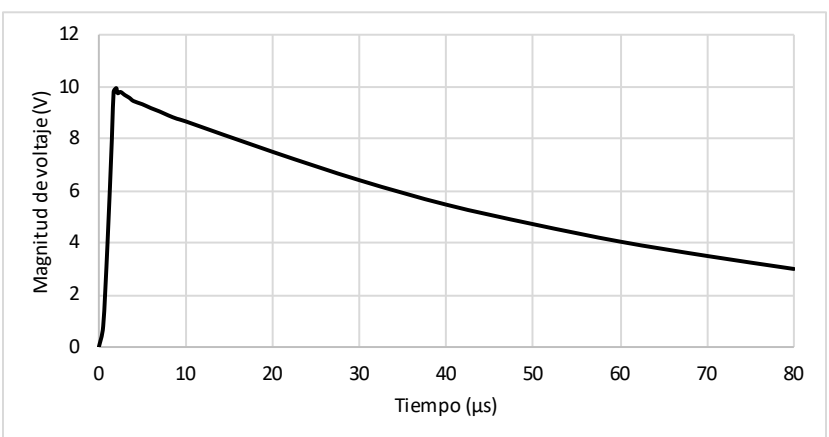

Figura 11. Señal de salida para la evaluación de la capacidad de aislamiento del resistor: impulso de $10 \mathrm{kV}$

Fuente: elaboración propia. 


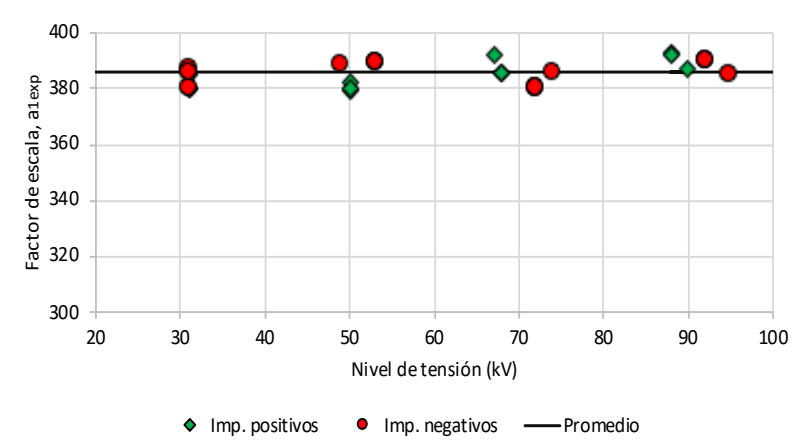

Figura 12. Gráfico de factor de escala rama \#1 vs. nivel de tensión

Fuente: elaboración propia.

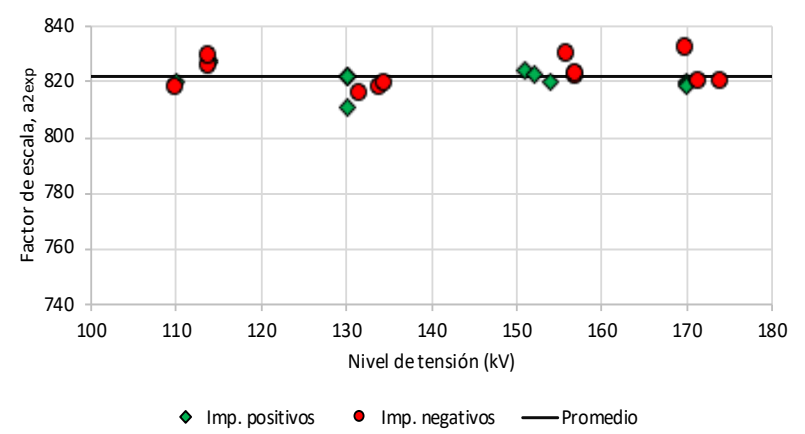

Figura 13. Gráfico de factor de escala rama \#2 vs. nivel de tensión

Fuente: elaboración propia.

Para verificar la validez de los factores de escala experimentales, se tomaron como referencia dos ensayos realizados en donde se aplicaron impulsos de 50 kV y 170 kV. En las tablas 3 y 4 se detaIlan los resultados.

Tabla 3. Datos para verificación del factor de escalarama \#1.

\begin{tabular}{lll}
\hline \multicolumn{1}{c}{ Parámetros } & \multicolumn{1}{c}{ Divisor resistivo } & \multicolumn{1}{c}{ DCA } \\
\hline Voltaje de salida & $50 \mathrm{~V}$ & $132 \mathrm{~V}$ \\
\hline Factor de escala & $1000: 1$ & $386: 1$ \\
\hline Voltaje de entrada & $50.000 \mathrm{~V}$ & $50.952 \mathrm{~V}$ \\
\hline
\end{tabular}

Fuente: elaboración propia.
Tabla 4. Datos para verificación del factor de escalarama \#2.

\begin{tabular}{lll}
\hline \multicolumn{1}{c}{ Parámetros } & \multicolumn{1}{c}{ Divisor resistivo } & \multicolumn{1}{c}{ DCA } \\
\hline Voltaje de salida & $170 \mathrm{~V}$ & $204 \mathrm{~V}$ \\
\hline Factor de escala & $1000: 1$ & $822: 1$ \\
\hline Voltaje de entrada & $170.000 \mathrm{~V}$ & $167.688 \mathrm{~V}$ \\
\hline
\end{tabular}

Fuente: elaboración propia.

Por medio de la ecuación (11) se calculan las diferencias porcentuales entre los voltajes máximos de las señales de impulso de los divisores resistivo y capacitivo amortiguado:

- Ensayo 1 (a $1 \exp =386: 1)$ : dif\%_1=2\%.

- Ensayo 2 (a2exp=822:1): dif\%_2=1\%.

Las diferencias porcentuales para los dos ensayos tomados como muestra, no superan el límite de variación de voltaje pico establecido por norma $\pm 3 \%$, de esta forma, para el análisis realizado experimentalmente, los factores de escala experimentales son válidos. La versión final del DCA de 300 kV y sus especificaciones técnicas, se muestran en la figura 14 y en la tabla 5 (Mina, 2013).

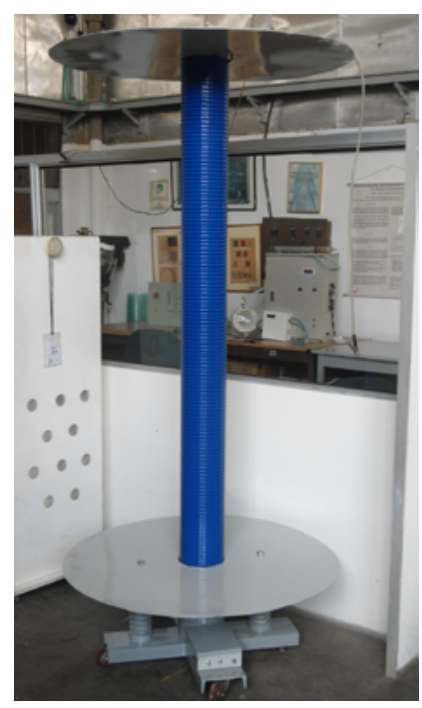

Figura 14. Divisor capacitivo amortiguado de $300 \mathrm{kV}$ (versión final)

Fuente: Laboratorio de Alta Tensión. Universidad del Valle. 
Tabla 5. Especificaciones técnicas del DCA de $300 \mathrm{kV}$ final.

\begin{tabular}{ll}
\hline Voltaje de entrada nominal & $300 \mathrm{kV}$ \\
\hline Voltaje de entrada máximo & $375 \mathrm{kV}$ \\
\hline Voltaje de salida nominal & $353 \mathrm{~V}$ \\
\hline Factores de escala & $386: 1$ \\
\hline Capacitancia de alto voltaje & $822: 1$ \\
\hline Capacitancia de bajo voltaje & $800 \mathrm{pF}$ \\
\hline Resistencia de amortiguamiento & $0,33 \mu \mathrm{F}$ \\
\hline Capacitancia parásita & $0,68 \mu \mathrm{F}$ \\
\hline Inductancia parásita & $479 \Omega$ \\
\hline Ancho de banda & $43 \mathrm{pF}$ \\
\hline Altura & $35 \mu \mathrm{H}$ \\
\hline Diámetro exterior de las placas (relajador de campo) & $2,1 \mathrm{MHz}$ \\
\hline
\end{tabular}

Fuente: elaboración propia.

\section{CONCLUSIONES}

Se presentó y aplicó una metodología para el diseño, construcción y evaluación de un divisor capacitivo amortiguado de 300 kV. Se utilizaron algunos componentes existentes de un divisor del Laboratorio de Alta Tensión de la Universidad del Valle.

La metodología desarrollada requirió del diseño y simulación de componentes del divisor a través de modelos eléctricos, los cuales facilitaron el ajuste de parámetros, por medio de herramientas computacionales, y su posterior verificación a través de ensayos eléctricos. Los modelos eléctricos propuestos en la metodología fueron suficientes para obtener resultados confiables en las simulaciones.

El resistor de amortiguamiento construido garantiza una impedancia puramente resistiva en un rango de frecuencia $20 \mathrm{~Hz}$ a $1 \mathrm{MHz}$.

Los factores de escala obtenidos experimentalmente son válidos dado que las diferencias porcentuales entre los voltajes máximos de las señales de impulso de los divisores resistivo y capacitivo amortiguado, no supera el límite de variación de voltaje pico establecido por norma $\pm 3 \%$.

\section{AGRADECIMIENTOS}

Los autores expresan un sincero agradecimiento al Laboratorio de Alta Tensión de la Universidad del Valle por su apoyo económico en el proyecto de grado "Rediseño de un prototipo de divisor de tensión capacitivo amortiguado de 300 kV para pruebas de tensión de impulso".

\section{REFERENCIAS}

Alfonso, A., Perdomo, L., Santamaría, F. y Gómez, C. (2014). Transient surges analysis in low voltage networks. Tecnura, 18(edición especial doctorado), 41-50. doi: https://doi. org/10.14483/22487638.9241

Daum, W. y Lynch, A.C. (1989). A high-voltage high-frequency voltage divider (for power line transients monitoring). IEE Proceedings A. Physical Science, Measurement and Instrumentation, Management and Education, 136(5), 254-255. doi: https://doi.org/10.1049/ ip-a-2.1989.0042

García, D.F. y Rincón, D.F. (2009). Diseño y construcción de un generador de impulso de voltaje de 10 kV. Tecnura, 12(24), 1-10. doi: https:// doi.org/10.14483/22487638.6262

García, D.F., Marlés-Sáenz, E., Prado, T.A. y Martínez, M. (2009). Metodology for Lightning Impulse Voltage Divisors Design. IEEE Latin America Transactions, 7(1), 71-77. doi: https://doi. org/10.1109/TLA.2009.5173467

IEEE Standard for High-Voltage Testing Techniques (2013). En IEEE Std 4-2013 (Revision of IEEE Std 4-1995), pp.1-213. doi: 10.1109/ ieeestd.2013.6515981.

International Electrotechnical Commission (IEC) (2010). IEC 60060-2 High-voltage Test Techniques, Part 2: Measuring Systems. Geneva, Switzerland. 
Kovačević, U., Brajović, D., Stanković, K. y Osmokrović, P. (2016). Measurement uncertainty of fast pulse voltages measurements with capacitive divider. En 2016 IEEE International Power Modulator and High Voltage Conference (IPMHVC), pp.v649-653. doi: https://doi. org/10.1109/IPMHVC.2016.8012783

Kuffel, E., Zaengl, W. y Kuffel, J. (2000). High Voltage Engineering- Fundamentals. 2a. ed. Toronto. Newnes. doi: https://doi.org/10.1016/ b978-075063634-6/50002-2

Mina, J.D. (2013). Rediseño de un prototipo de divisor de tensión capacitivo amortiguado de $300 \mathrm{kV}$ para pruebas de tensión de impulso. Cali: Universidad del Valle, Biblioteca Digital. doi: https://doi.org/10.22395/rium. v13n25a6

Ortiz, A.J., Jiménez, R. y Ramos, O.L. (2014). Inspección de calidad para un sistema de producción industrial basado en el procesamiento de imágenes. Tecnura, 18(41), 76-90. doi: https://doi.org/10.14483/udistrital.jour. tecnura.2014.3.a06

Pan, Y., Shao, H., Xiao, D., Lai, L., Shi, L., Zhou, L. y Zhao, W. (2015). Development of 300-kV Air-Insulation Standard Impulse Measurement System. IEEE Transactions on Instrumentation and Measurement, 64(6), 1627-1635. doi: https://doi.org/10.1109/tim.2015.2408799

Passon, S., Gitin, I. y Meisner, J. (2016). Investigating the properties of precision resistors for the application in high voltage DC dividers. En 2016 IEEE International Workshop on Applied Measurements for Power Systems (AMPS) (pp. 1-6). doi: https://doi.org/10.1109/ amps.2016.7602872
Pattanadech, N., Potivetkul, S. y Yuttagowith, P. (2006). Corona Phenomena of Various High Voltage Shielding Types. En 2006 International Conference on Power System Technology (pp. 1-6). Chongqing. doi: https://doi.org/10.1109/ icpst.2006.321552

Pattarakijkul, D., Kurupakorn, C. y Charoensook, A. (2010). Construction and evaluation of 100 kV DC high voltage divider. En CPEM 2010 (pp. 677-679). doi: https://doi.org/10.1109/ cpem.2010.5543420

Prochazka, R., Hlavacek, J., Knenicky, M. y Mahmoud, R. (2016). Determination of frequency characteristics of high voltage dividers in frequency domain. En 2016 17th International Scientific Conference on Electric Power Engineering (EPE) (pp. 1-4). doi: https://doi. org/10.1109/epe.2016.7521821

Teachawong, M. y Trakulkan, C. (2014). Design and invention a $150 \mathrm{kV}$ capacitive high voltage divider set. En The 4th Joint International Conference on Information and Communication Technology, Electronic and Electrical Engineering (JICTEE) (pp. 1-5). doi: https://doi. org/10.1109/jictee.2014.6804114

Vera, M.M. y Prado, T. (2006). Diseño y construcción de un divisor de tensión capacitivo amortiguado de 300 kV. Cali: Universidad del Valle, Biblioteca Digital

Yan, W., Zhao, W. y Li, Y. (2018). Effect of Step Response Measurement Arrangement on the Correction of Lightning Impulses Measured with Ultra-High-Voltage Dividers. En 2018 Conference on Precision Electromagnetic Measurements (CPEM 2018) (pp. 1-2). doi: https://doi. org/10.1109/cpem.2018.8501145

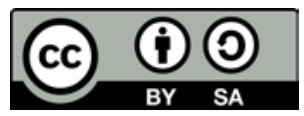

